\title{
Poisson's Theory for Analysis of Bending of Isotropic and Anisotropic Plates
}

\author{
K. Vijayakumar \\ Department of Aerospace Engineering, Indian Institute of Science, Bangalore 560 012, India \\ Correspondence should be addressed to K. Vijayakumar; kazavijayakumar@gmail.com
}

Received 30 May 2013; Accepted 19 June 2013

Academic Editors: M. Garg and D. Huang

Copyright (C) 2013 K. Vijayakumar. This is an open access article distributed under the Creative Commons Attribution License, which permits unrestricted use, distribution, and reproduction in any medium, provided the original work is properly cited.

\begin{abstract}
Sixteen-decade-old problem of Poisson-Kirchhoff's boundary conditions paradox is resolved in the case of isotropic plates through a theory designated as "Poisson's theory of plates in bending." It is based on "assuming" zero transverse shear stresses instead of strains. Reactive (statically equivalent) transverse shear stresses are gradients of a function (in place of in-plane displacements as gradients of vertical deflection) so that reactive transverse stresses are independent of material constants in the preliminary solution. Equations governing in-plane displacements are independent of the vertical (transverse) deflection $w_{0}(x, y)$. Coupling of these equations with $w_{0}$ is the root cause for the boundary conditions paradox. Edge support condition on $w_{0}$ does not play any role in obtaining in-plane displacements. Normally, solutions to the displacements are obtained from governing equations based on the stationary property of relevant total potential and reactive transverse shear stresses are expressed in terms of these displacements. In the present study, a reverse process in obtaining preliminary solution is adapted in which reactive transverse stresses are determined first and displacements are obtained in terms of these stresses. Equations governing second-order corrections to preliminary solutions of bending of anisotropic plates are derived through application of an iterative method used earlier for the analysis of bending of isotropic plates.
\end{abstract}

\section{Introduction}

Kirchhoff's theory [1] and first-order shear deformation theory based on Hencky's work [2] abbreviated as FSDT of plates in bending are simple theories and continuously used to obtain design information. Kirchhoff's theory consists of a single variable model in which in-plane displacements are expressed in terms of gradients of vertical deflection $w_{0}(x, y)$ so that zero face shear conditions are satisfied. $w_{0}$ is governed by a fourth-order equation associated with two edge conditions instead of three edge conditions required in a 3D problem. Consequence of this lacuna is the wellknown Poisson-Kirchhoff boundary conditions paradox (see Reissner's article [3]).

Assumption of zero transverse shear strains is discarded in FSDT forming a three-variable model. Vertical deflection $w_{0}(x, y)$ and in-plane displacements $[u, v]=$ $z\left[u_{1}(x, y), v_{1}(x, y)\right]$ are coupled in the governing differential equations and boundary conditions. Reactive (statically equivalent) transverse shears are combined with in-plane shear resulting in approximation of associated torsion problem instead of flexure problem. In Kirchhoff's theory, inplane shear is combined with transverse shear implied in Kelvin and Tait's physical interpretation of contracted boundary condition [4]. In fact, torsion problem is associated with flexure problem whereas flexure problem (unlike directly or indirectly implied in energy methods) is independent of torsion problem. Reissner [3] in his article felt that inclusion of transverse shear deformation effects was presumed to be the key in resolving Poisson-Kirchhoff's boundary conditions paradox. Recently, it is shown that the second-order correction to $w_{0}(x, y)$ by either Reissner's theory or FSDT corresponds to approximate solution of a torsion problem [5]. Coupling between bending and associated torsion problems is eliminated earlier [6] by using zero rotation $\omega_{z}=\left(v_{, x}-u_{, y}\right)$ about the vertical axis. The proposal of zero $\omega_{z}$ does not, however, resolve the paradox in a satisfactory manner. In the reference [6], face deflection $w_{0}$ in (8) is from zero face 
shear conditions whereas neutral plane deflectio $n w_{0}$ from edge support condition is associated with self-equilibrating transverse shear stresses.

The condition $\omega_{z}=0$ decoupling the bending and torsion problems is satisfied in Kirchhoff's theory. If this condition is imposed in FSDT, sum of the strains $\left(\varepsilon_{x}+\varepsilon_{y}\right)$ in the isotropic plate is governed by a second-order equation due to applied transverse loads. Reactive transverse shear stresses are in terms of gradients of $\left(\varepsilon_{x}+\varepsilon_{y}\right)$ uncoupled from $w_{0}$. Thicknesswise linear distribution of $\sigma_{z}$ is zero from the equilibrium equation governing transverse stresses. Normal strain $\epsilon_{z}$ from constitutive relation is linear in $z$ in terms of $\left(\varepsilon_{x}+\varepsilon_{y}\right)$. Reactive transverse shear stresses and thickness-wise linear strain $\varepsilon_{z}$ form the basis for resolving the paradox and for obtaining higher order corrections to the displacements. The theory thus developed is designated as "Poisson's theory of plates in bending".

The previously mentioned Poisson's theory is applied to the analysis of anisotropic plates using reactive transverse shear stresses as gradients of a function. Normally, solutions to the displacements are obtained from governing equations based on stationary property of relevant total potential and the reactive transverse shear stresses are expressed in terms of these displacements. In the present work, reverse process is adapted in which reactive transverse stresses are determined first and the displacements are obtained in terms of these stresses. Equations governing second-order corrections to the preliminary solutions are derived through the application of an iterative method used earlier [7] for the analysis of bending of isotropic plates.

\section{Equations of Equilibrium and Edge Conditions}

For simplicity in presentation, a rectilinear domain bounded by $0 \leq X \leq a, 0 \leq Y \leq b, Z= \pm h$, with reference to Cartesian coordinate system $(X, Y, Z)$ is considered. For convenience, coordinates $X, Y, Z$ and displacements $U, V, W$ in nondimensional form $x=X / L, y=Y / L, z=$ $Z / h, u=U / h, v=V / h, w=W / h$ and half-thickness ratio $\alpha=h / L$ with reference to a characteristic length $L$ in $X-Y$ plane are used ( $L$ is defined such that mod of $x$ and $y$ are equal to or less than 1). With the previous notation and $\Leftrightarrow$ indicating interchange, equilibrium equations in terms of stress components are

$$
\begin{gathered}
\alpha\left(\sigma_{x, x}+\tau_{x y, y}\right)+\tau_{x z, z}=0 \Longleftrightarrow(x, y), \\
\alpha\left(\tau_{x z, x}+\tau_{y z, y}\right)+\sigma_{z, z}=0,
\end{gathered}
$$

in which suffix after "," denotes partial derivative operator.

Edge conditions are prescribed such that $\left(w, \tau_{x z}, \tau_{y z}\right.$, $\left.\gamma_{x z}, \gamma_{y z}\right)$ are even in $z$, and $\left(u, v, \sigma_{x}, \sigma_{y}, \tau_{x y}, \varepsilon_{x}, \varepsilon_{y}, \gamma_{x y}, \sigma_{z}, \varepsilon_{z}\right)$ are odd in $z$. In the primary flexure problem, the plate is subjected to asymmetric load $\sigma_{z}= \pm q(x, y) / 2$ and zero shear stresses along $z= \pm 1$ faces. Three conditions to be satisfied along $x$ (and $y$ ) constant edges are prescribed in the form

$$
\begin{array}{r}
u=0 \text { or } \sigma_{x}=z T_{x}(y) \Longleftrightarrow(x, y),(u, v), \\
v=0 \text { or } \tau_{x y}=z T_{x y}(y) \Longleftrightarrow(x, y),(u, v), \\
w=0 \text { or } \tau_{x z}=\left(\frac{1}{2}\right)\left(1-z^{2}\right) T_{x z}(y) \Longleftrightarrow(x, y) .
\end{array}
$$

\section{Stress-Strain and Strain-Displacement Relations}

In displacement based models, stress components are expressed in terms of displacements, via six stress-strain constitutive relations and six strain-displacement relations. In the present study, these relations are confined to the classical small deformation theory of elasticity.

It is convenient to denote displacement and stress components of anisotropic plates as

$$
\begin{gathered}
{[u, v, w]=\left[u_{i}\right], \quad\left[\sigma_{x}, \sigma_{y}, \tau_{x y}\right]=\left[\sigma_{i}\right] \quad(i=1,2,3),} \\
{\left[\tau_{x z}, \tau_{y z}, \sigma_{z}\right]=\left[\sigma_{i}\right] \quad(i=4,5,6) .}
\end{gathered}
$$

Strain-stress relations in terms of compliances $\left[S_{i j}\right]$ with the usual summation convention are

$$
\varepsilon_{i}=S_{i j} \sigma_{j}, \quad \varepsilon_{r}=S_{r s} \sigma_{s}, \quad(i, j=1,2,3,6) \quad(r, s=4,5) .
$$

We have from semi-inverted strain-stress relations with $\left[Q_{i j}\right]$ denoting inverse of $\left[S_{i j}\right]$ :

$$
\sigma_{i}=Q_{i j}\left(\varepsilon_{j}-S_{6 j} \sigma_{z}\right) \quad(i, j=1,2,3) .
$$

Normal strain $\varepsilon_{z}$ is given by

$$
\varepsilon_{z}=S_{6 j} \sigma_{j}, \quad j=1,2,3,6 .
$$

Transverse shear strains with $\left[Q_{r s}\right]$ denoting inverse of $\left[S_{r s}\right]$ are

$$
\varepsilon_{r}=Q_{r s} \sigma_{s}, \quad(r, s=4,5) .
$$

Strains $\varepsilon_{i}$ from strain-displacement relations are

$$
\begin{gathered}
{\left[\varepsilon_{1}, \varepsilon_{2}, \varepsilon_{3}\right]=\alpha\left[u_{, x}, v_{, y}, u_{, y}+v_{,_{x}}\right],} \\
{\left[\varepsilon_{4}, \varepsilon_{5}, \varepsilon_{6}\right]=\left[u+\alpha w_{, x}, v+\alpha w_{, y}, w_{, z}\right] .}
\end{gathered}
$$

\section{4. $f_{n}(z)$ Functions and Their Use}

We use thickness-wise distribution functions $f_{n}(z)$ generated from recurrence relations [7] with $f_{0}=1, f_{2 n+1, z}=f_{2 n}$, $f_{2 n+2, z}=-f_{2 n+1}$ such that $f_{2 n+2}( \pm 1)=0$. They are (up to $n=5)$

$$
\begin{gathered}
{\left[f_{1}, f_{2}, f_{3}\right]=\left[z, \frac{\left(1-z^{2}\right)}{2}, \frac{\left(z-z^{3} / 3\right)}{2}\right],} \\
{\left[f_{4}, f_{5}\right]=\left[\frac{\left(5-6 z^{2}+z^{4}\right)}{24}, \frac{\left(25 z-10 z^{3}+z^{5}\right)}{120}\right] .}
\end{gathered}
$$


Displacements, strains, and stresses are expressed in the form $(\operatorname{sum} n=0,1,2,3, \ldots)$

$$
\begin{gathered}
{[w, u, v]=\left[f_{2 n} w_{2 n}, f_{2 n+1} u_{2 n+1}, f_{2 n+1} v_{2 n+1}\right],} \\
{\left[\varepsilon_{x}, \varepsilon_{y}, \gamma_{x y}, \varepsilon_{z}\right]=f_{2 n+1}\left[\varepsilon_{x}, \varepsilon_{y}, \gamma_{x y}, \varepsilon_{z}\right]_{2 n+1} .}
\end{gathered}
$$

(Note that $w_{2 n}=-\varepsilon_{z 2 n-1}, n \geq 1$ )

$$
\begin{aligned}
& {\left[\sigma_{x}, \sigma_{y}, \tau_{x y}, \sigma_{z}\right]=f_{2 n+1}\left[\sigma_{x}, \sigma_{y}, \tau_{x y}, \sigma_{z}\right]_{2 n+1},} \\
& {\left[\gamma_{x z}, \gamma_{y z}, \tau_{x z}, \tau_{y z}\right]=f_{2 n}\left[\gamma_{x z}, \gamma_{y z}, \tau_{x z}, \tau_{y z}\right] .}
\end{aligned}
$$

In the previous equations, variables associated with $f(z)$ functions are functions of $(x, y)$ only.

\section{Sequence of Trivially Known Steps without Any Assumptions in Preliminary Analysis}

(a) Due to prescribed zero $\left(\tau_{x z}, \tau_{y z}\right)$ along $z= \pm 1$ faces of the plate, $\left(\tau_{x z 0}, \tau_{y z 0}\right) \equiv 0$ in the plate.

(b) $\left(\gamma_{x z 0}, \gamma_{y z 0}\right) \equiv 0$ from constitutive relations (5).

(c) Static equilibrium equation (2) gives $\sigma_{z 1} \equiv 0$.

(d) In the absence of $\left(u_{1}, v_{1}\right),\left(\varepsilon_{x 1}, \varepsilon_{y 1}, \gamma_{x y 1}\right) \equiv 0$ from strain-displacement relations (8).

(e) $\left(\sigma_{x 1}, \sigma_{y 1}, \tau_{x y 1}\right) \equiv 0$ from constitutive relations (6).

(f) $\varepsilon_{z 1} \equiv 0$ from relation (7).

(g) $w=w_{0}(x, y)$ from thickness-wise integration of $\varepsilon_{z}=$ 0 .

(h) From steps (b) and (g), integration of $\left[u_{, z}+\alpha w_{, x}, v_{, z}+\right.$ $\left.\alpha w_{, y}\right]=[0,0]$ in the thickness direction gives $[u, v]=$ $-z \alpha\left[w_{0, x}, w_{0, y}\right]$.

In the present study as in the earlier work [6], $[u, v]$ linear in $z$ are unknown functions as in FSDT. From integration of $\left[u_{, z}+\alpha w_{, x}, v_{, z}+\alpha w_{, y}\right]=[0,0]$ in the face plane instead of thickness direction, one gets vertical deflection $w_{0}(x, y)$ in the form

$$
\alpha w_{0}=-\int\left[u_{1} d x+v_{1} d y\right]
$$

Since $w_{0}$ is from satisfaction of zero face shear conditions, it can be considered as face deflection $w_{0 F}$ though it is same for all face parallel planes (note that prescribed zero $w_{0}$ along a segment of the neutral plane implies zero $w_{0}$ along the corresponding segment of the intersection of face plane with wall of the plate since $w_{0}$ is independent of $z$ and vice versa). It is analytic in the domain of the plate if $\omega_{z}$ is zero. In such a case, we note from strain-displacement relations (8) that

$$
\left[\varepsilon_{1, y}, \varepsilon_{2, x}, \varepsilon_{3, y}, \varepsilon_{3, x}\right]=\alpha^{2}\left[v_{x_{x x}}, u_{, y y}, 2 u_{, y y}, 2 v_{, x x}\right] \text {. }
$$

(i) In the absence of applied transverse loads, integrations of (1), (2) using Kirchhoff's displacements give a homogeneous fourth-order equation governing $w_{0}$. In the present analysis as in FSDT, static equations governing $\left[u_{1}, v_{1}\right]$ are, however, given by

$$
Q_{1 i} \varepsilon_{i, x}+Q_{3 i} \varepsilon_{i, y}=0, \quad Q_{2 i} \varepsilon_{i, y}+Q_{3 i} \varepsilon_{3 i, x}=0 \quad(i=1,2,3) .
$$

They are subjected to the edge conditions (3a), (3b) along $x$ (and $y$-) constant edges.

Equations (15) governing $\left[u_{1}, v_{1}\right]$ are uncoupled due to relations (14) in the case of isotropic plates whereas they are coupled without cross-derivatives $\left[u_{1 x y}, v_{1 x y}\right]$ in the case of anisotropic plates. In the absence of prescribed bending stress all along the closed boundary of the plate, in-plane $\tau_{x y}$ distribution corresponds to pure torsion where as it is different in the presence of bending load. These $\tau_{x y}$ distributions are discussed later in Section 6.2.

(j) In FSDT, (15) correspond to neglecting shear energy from transverse shear deformations. Vertical deflection $w_{0}$ has to be obtained from (14) using the solutions for $\left[u_{1}, v_{1}\right]$ from (15). Reactive transverse stresses are expressed in terms of $\left[u_{1}, v_{1}\right]$. They are dependent on material constants different from prescribed nonzero transverse shear stresses along the edges of the plate.

(k) In the development of Poisson's theory, (15) are coupled with reactive transverse stresses.

\section{Poisson's Theory}

In the preliminary solution, steps (a)-(g) in Section 5 are unaltered. That is, transverse stresses and strains are zero and $w=w_{0}(x, y)$. In the absence of higher order in-plane displacement terms, reactive transverse shear stresses are parabolic and gradients of a function $\psi(x, y)$, that is, inplane distributions of transverse shear stresses $\left[\tau_{x z}, \tau_{y z}\right]=$ $\alpha\left[\psi_{2, x}, \psi_{2, y}\right]$. Equation governing $\psi\left(=\psi_{2}\right)$ from thicknesswise integration of (2) is

$$
\alpha^{2} \Delta \psi+\sigma_{z 3}=0
$$

In the previous equation, $\sigma_{z 3}$ is coefficient of $f_{3}(z)$. Satisfaction of the load condition $\sigma_{z}= \pm q / 2$ along $z= \pm 1$ faces gives $\sigma_{z 3}=(3 / 2) q$ so that

$$
\alpha^{2} \Delta \psi+\left(\frac{3}{2}\right) q=0
$$

Edge condition on $\psi$ is either $\psi=0$ or its outward normal gradient is equal to the prescribed shear stress along each segment of the edge. Note that the transverse stresses thus obtained are independent of material constants. In the isotropic plate, $\psi(x, y)=E^{\prime} e_{1}$ in which $E^{\prime}=E /\left(1-v^{2}\right)$ and $e_{1}=\left(\varepsilon_{x 1}+\varepsilon_{y 1}\right)$.

Integrated equilibrium equations (1) in terms of in-plane strains are $(\operatorname{sum} j=1,2,3)$ :

$$
\begin{aligned}
& {\left[Q_{1 j} \varepsilon_{j, x}+Q_{3 j} \varepsilon_{j, y}\right]-\tau_{x z 2}=0,} \\
& {\left[Q_{2 j} \varepsilon_{j, y}+Q_{3 j} \varepsilon_{j, x}\right]-\tau_{y z 2}=0 .}
\end{aligned}
$$

By substituting strains in terms of $\left[u_{1}, v_{1}\right],(18)$ are two equations governing $\left(u_{1}, v_{1}\right)$ and have to be solved with conditions along $x$ - (and $y$-) constant edge

$$
\begin{gathered}
u=0 \text { or } \sigma_{x}=z T_{x}(y) \Longleftrightarrow(x, y),(u, v), \\
v=0 \text { or } \tau_{x y}=z T_{x y}(y) \Longleftrightarrow(x, y),(u, v) .
\end{gathered}
$$


Vertical deflection $w_{0}$ is given by (13). As mentioned earlier, cross-derivatives of $\left[u_{1}, v_{1}\right]$ do not exist in (18), (19a), and (19b) due to (14). In the isotropic case, (18) are uncoupled and are simply given by

$$
\alpha^{2} \Delta u_{1}=\alpha e_{1, x} \Longleftrightarrow(x, y),(u, y) .
$$

Edge conditions (19a) and (19b) are also uncoupled and they are given by

$$
\begin{gathered}
u_{1}=0 \text { or } E^{\prime}(1-v) \alpha u_{1, x}=T_{x}(y) \Longleftrightarrow(x, y),(u, v), \\
v_{1}=0 \text { or } 2 G \alpha v_{1, x}=T_{x y}(y) \Longleftrightarrow(x, y),(u, v) .
\end{gathered}
$$

The condition $\psi_{0}=0$ in solving (17) is different from the usual condition $w_{0}=0$ in the Kirchhoff's theory and FSDT. The function $\psi_{0}$ is related to the normal strain $\varepsilon_{z}$. In the isotropic case, $\psi_{0}$ is proportional to $e_{1}(x, y)=\left(\varepsilon_{x 1}+\varepsilon_{y 1}\right)$. Gradients of $\psi_{0}$ are proportional to gradients of transverse strains in FSDT. If the plate is free of applied transverse stresses, that is, the plate is subjected to bending and twisting moments only, $e_{1}$ is proportional to $\Delta w_{0}$. The function $\psi_{0}(x, y)$, thereby, $\Delta w_{0}$ is identically zero from (2) (in Kirchhoff's theory, the tangential gradient of $\Delta w_{0}$ is proportional to the corresponding gradient of applied $\tau_{x y}$ along the edge of the plate implied from Kelvin and Tait's physical interpretation of the contracted transverse shear condition). This Laplace equation is not adequate to satisfy two in-plane edge conditions. One needs its conjugate harmonic functions to express in-plane displacements in the form

$$
\left[u_{1}, v_{1}\right]=-\alpha z\left[w_{0, x}+\varphi_{0, y}, w_{0, y}-\varphi_{0, x}\right] .
$$

The function $\varphi_{0}$ was introduced earlier by Reissner [8] as a stress function in satisfying (2). Note that the two variables $u_{1}$ and $v_{1}$ are expressed in terms of gradients of two functions $w_{0}$ and $\varphi_{0}$.

After finding $w_{0}$ and $\varphi_{0}$ from solving in-plane equilibrium equations (1), correction $w_{0 c}$ to $w_{0}$ from zero face shear conditions is given by

$$
w_{0 c}=\int\left[\phi_{0, y} d x+\phi_{0, x} d y\right]
$$

One should note here that $w(x, y)=w_{0}+w_{0 c}$ does not satisfy prescribed edge condition on $w$.

In FSDT, $\left[u_{1}, v_{1}\right]$ are obtained from solving (1). There is no provision to find $w_{0}$ and it has to be obtained from zero face shear conditions in the form

$$
\alpha w_{0}=-\int\left[u_{1} d x+v_{1} d y\right] .
$$

If the applied transverse stresses are nonhomogeneous along a segment of the edge, $w_{0}$ is coupled with $\left[u_{1}, v_{1}\right]$ in (1) and (2) and edge conditions (3a), (3b), and (3c).

With reference to the present analysis, it is relevant to note the following observation: in the preliminary solution, $\sigma_{z}, \alpha\left[\tau_{x z}, \tau_{y z}\right], \alpha^{2}\left[\sigma_{x}, \sigma_{y}, \tau_{x y}\right]$ are of $O(1)$. As such, estimation of in-plane stresses, thereby, in-plane displacements is not dependent on $w_{0}$. The support condition $w_{0}=0$ along the edge of the plate does not play any role in determining the in-plane displacements.
6.1. Comparison with Analysis of Extension Problems. It is interesting to compare the previous analysis with that of extension problem. In extension problem, applied shear stresses along the faces of the plate are gradients of a potential function and they are equivalent to linearly varying body forces, independent of elastic constants, in the in-plane equilibrium equations. Rotation $\omega_{z} \neq 0$ (but $\Delta \omega_{z}=0$ ) and equations governing displacements $\left[u_{0}, v_{0}\right]$ are coupled. Also, vertical deflection is given by thickness-wise integration of $\varepsilon_{z 0}$ from constitutive relation and higher order corrections are dependent on this vertical deflection. In the present analysis of bending problems, in-plane distributions of transverse shear stresses are gradients of a function related to applied edge transverse shear and face normal load through equilibrium equation (2). They are also independent of elastic constants and derivative $f_{2, z}$ of parabolic distribution of each of them is equivalent to body force in linearly varying inplane equilibrium equations. Vertical deflection $w_{0}$ is purely from satisfaction of zero shear stress conditions along faces of the plate. Higher order corrections are dependent on normal strains and independent of $w_{0}$. It can be seen that steps in the analysis of bending problem are complementary to those in the classical theory of extension problem.

6.2. Associated Torsion Problems. First-order shear deformation theory and higher order shear deformation theories do not provide proper corrections to initial solution (from Kirchhoff's theory) of primary flexure problems. In these theories, corrections are due to approximate solutions of associated torsion problems. In fact, one has to have a relook at the use of shear deformation theories based on plate (instead of 3D) element equilibrium equations other than Kirchhoff's theory in the analysis of flexure problems. In the earlier work [5], we have mentioned that this coupling of bending and torsion problems is nullified in the limit of satisfying all equations in the $3 \mathrm{D}$ problems. However, these higher order theories in the case of primary flexure problem defined from Kirchhoff's theory are with reference to finding the exact solution of associated torsion problem only. In fact, one can obtain exact solution of the torsion problem (instead of using higher order polynomials in $z$ by expanding $z$ and $f_{3}(z)$ in sine series and $f_{2}(z)$ in cosine series. In a pure torsion problem, normal stresses and strains are zero implying that

$$
[u, v, w]=[u(y, z), v(x, z), w(x, y)]
$$

Rotation $\omega_{z} \neq 0$ and warping displacements $(u, v)$ are independent of vertical displacement $w$. In FSDT, $\omega_{z} \neq 0$ and corrections to Kirchhoff's displacements are due to the solution of approximate torsion problem. In the isotropic rectangular plate, warping function $u(y, z)$ is harmonic in $y$ $z$-plane. It has to satisfy $G \alpha u_{, y}=T_{u}(y, z)$ with prescribed $T_{u}$ along an $x=$ constant edge. By expressing $u$ in product form $u=f_{u}(y) g_{u}(z)$, we have

$$
\alpha^{2} f_{u, y y} g_{u}+f_{u} g_{u, z z}=0 \text {. }
$$


By taking $g_{u n}=\sin \lambda_{n} z$ where $\lambda_{n}=(2 n+1) \pi / 2$ satisfying zero face shear conditions, we get

$$
f_{u n}=A_{u n} \cosh \left(\frac{\lambda_{n}}{\alpha}\right) y+B_{u n} \sinh \left(\frac{\lambda_{n}}{\alpha}\right) y .
$$

If prescribed $T_{u}$ is $f_{u n}$ with specified constants $\left(A_{u n}, B_{u n}\right)$, clearly there is no provision to satisfy zero in-plane shear condition along $y=$ constant edges. It has to be nullified with corresponding solution from bending problem. That is why a torsion problem is associated with bending problem but not vice versa. In a corresponding bending problem, all stress components are zero except $\tau_{x y}=-f_{u n}(y) g_{u n}(z)$. This solution is used only in satisfying edge condition along $y=$ constant edges in the presence of specified $T_{u n}$ along $x=$ constant edges. It shows that $\tau_{x y}$ distribution in flexure problem is nullified in the limit in shear deformation theories due to torsion.

It is interesting to note that the previous mentioned deficiency, due to coupling with $w_{0}$ in the plate element equilibrium equations in FSDT and higher order shear deformation theories, does not exist if applied $\tau_{x y}$ is zero all along the closed boundary of the plate. It is complementary to the fact that boundary condition paradox in Kirchhoff's theory does not exist if tangential displacement and $w_{0}$ are zero all along the boundary of the plate.

6.3. Illustrative Example: Simply Supported Isotropic Square Plate. Consider a simply supported isotropic square plate subjected to vertical load

$$
q=q_{0} \sin \left(\frac{\pi x}{a}\right) \sin \left(\frac{\pi y}{a}\right)
$$

Exact values for neutral plane and face deflections obtained earlier [7] with $v=0.3, \alpha=(1 / 6)$ are

$$
\begin{aligned}
& \left(\frac{E}{2 q_{0}}\right) w\left(\frac{a}{2}, \frac{a}{2}, 0\right)=4.487, \\
& \left(\frac{E}{2 q_{0}}\right) w\left(\frac{a}{2}, \frac{a}{2}, 1\right)=4.166 .
\end{aligned}
$$

In this example, Poisson-Kirchhoff's boundary conditions paradox does not exist. Hence, Poisson's theory gives the same solution obtained in the author's previous mentioned work. Concerning face value in (30), Kirchhoff's theory gives a value of 2.27 that is same for all face parallel planes. Poisson's theory gives an additional value of 0.267 attributable to $\varepsilon_{z 1}$ and it is same for neutral plane deflection since $w(x, y, z)$ can be expressed as

$$
\begin{aligned}
w(x, y, z) & =w_{0 F}(x, y)+f_{2}(z) w_{2} \\
& =w_{0 N}(x, y)-\left(\frac{1}{2}\right) z^{2} \varepsilon_{z 1} .
\end{aligned}
$$

In the previous expression, $w_{0 N}$ is deflection of the neutral plane. Higher order correction to $w_{0}$ uncoupled from torsion is 1.262 so that total correction to the value from Kirchhoff's theory is about 1.53 [7]. Correction due to coupling with torsion is 1.45 [5] whereas it is 1.423 from FSDT and other sixth order theories [9]. With reference to numerical values reported by Lewiński [9], the previous correction is 1.412 in Reddy's 8th-order theory and less than 1.23 in Reissner's 12 thorder and other higher order theories. It clearly shows that these shear deformation theories do not lead to solutions of bending problems (note that the value $(2.27+1.423)$ from FSDT corresponds to neutral plane deflection and it is in error by about $17.7 \%$ from the exact value).

In view of the previous observations, it is relevant to make the following remarks: physical validity of $3 \mathrm{D}$ equations is constrained by limitations of small deformation theory. For $\alpha=1$, this constraint is dependent on material constants, geometry of the domain, and applied loads. Range of validity of $2 \mathrm{D}$ plate theories arises for small values of $\alpha$. Kirchhoff's theory gives lower bound for this $\alpha$. For this value of $\alpha$ and for slightly higher values of $\alpha$ than this lower bound, Kirchhoff's theory is used, in spite of its known deficiencies, due to its simplicity to obtain design information. Similarly, FSDT and other sixth-order shear deformation theories are used for some additional range of values of $\alpha$ due to the corrections over Kirchhoff's theory though these corrections are due to coupling with approximate torsion problems. They serve only in giving guidance values for applicable design parameters for small range of values of $\alpha$ beyond its lower bound. They do not provide initial set of equations in formulating proper sequence of sets of $2 \mathrm{D}$ problems converging to the $3 \mathrm{D}$ problem. As such, comparison of solutions from Kirchhoff theory and FSDT with the corresponding solutions in the present analysis does not serve much purpose.

Since $w_{0 N}$ has to be higher than $w_{0 F}$, one has to obtain the correction to $w_{0 N}$ before finding correction to internal distribution of $w(x, y, z)$ along with correction to $w_{0 F}$. Initial correction to $w_{0 N}$ is obtained earlier [7] in the form

$$
w=w_{0}-\left[f_{2}(0) \varepsilon_{z 1}+f_{4}(0) \varepsilon_{z 3}\right] .
$$

It is the same for face deflection (note that $w_{2}$ in the previously mentioned author's work is required only to obtain $\left.\varepsilon_{z 3}\right)$. As such, $w_{0 N}$ is further corrected from solution of a supplementary problem. This total correction over face deflection is about 0.658 giving a value of 4.458 which is very close to the exact value 4.487 in (29). However, we note that the error in the estimated value $(=3.8)$ of $w_{0 F}$ is relatively high compared to the accuracy achieved in the neutral plane deflection. It is possible to improve estimation of $w_{0 F}$ by including $\varepsilon_{z 1}$ in $\left(u_{3}, v_{3}\right)$ such that $\left(\tau_{x z 2}, \tau_{y z 2}\right)$ are independent of $\varepsilon_{z 1}$. In the present example, correction to face deflection changes to

$$
\left(\frac{E}{2 q_{0}}\right) w_{\text {face } \max }=\frac{((6 / 5)((1+v) /(1-v))) \beta^{2}}{((2 / 5)((4-v) /(1-v))) \beta^{2}-1}
$$

In the previous equation, $\beta^{2}=2 w_{0 F} \alpha^{2} \pi^{2}$. Correction $\varepsilon_{z 3}$ (= $1.262)$ to face value changes to 1.431 giving $3.97(=2.27+$ $0.267+1.431$ ) for face deflection which is under $4.7 \%$ from exact value. 


\section{Anisotropic Plate: Second-Order Corrections}

We note that $\varepsilon_{z 1}$ does not participate in the determination of $\left(u_{1}, v_{1}\right)$ and it is obtained from the constitutive relation (6) in the interior of the plate. It is zero along an edge of the plate if $w=0$ is specified condition. With zero transverse shear strains, specification of $\varepsilon_{z 1}=0$ instead of $w_{0}=0$ is more appropriate along a supported edge since $w_{0}=0$ implies zero tangential displacement along a straight edge which is the root cause of Poisson-Kirchhoff's boundary conditions paradox. As such, edge support condition on $w$ does not play any role in obtaining the in-plane displacements and reactive transverse stresses.

In the first stage of iterative procedure, second-order reactive transverse stresses have to be obtained by considering higher order in-plane displacement terms $u_{3}$ and $v_{3}$. However, one has to consider limitation in the previous Poisson's theory like in Kirchhoff's theory; namely, reactive $\sigma_{z}$ is zero at locations of zeros of $q$. It is identically zero in higher order approximations since $f_{2 n+1}(z)$ functions are not zero along $z= \pm 1$ faces. Moreover, it is necessary to account for its dependence on material constants. To overcome these limitations, it is necessary to keep $\sigma_{z 5}$ as a free variable by modifying $f_{5}$ in the form

$$
f_{5}^{*}(z)=f_{5}(z)-\beta_{3} f_{3}(z) .
$$

In the previous equation, $\beta_{3}=\left[f_{5}(1) / f_{3}(1)\right]$ so that $f_{5}^{*}( \pm 1)=$ 0 . Denoting coefficient of $f_{3}$ in $\sigma_{z}$ by $\sigma_{z 3}^{*}$, it becomes

$$
\sigma_{z 3}^{*}=\sigma_{z 3}-\beta_{3} \sigma_{z 5} \text {. }
$$

Displacements $\left(u_{3}, v_{3}\right)$ are modified such that they are corrections to face parallel plane distributions of the preliminary solution and are free to obtain reactive stresses $\tau_{x z 4}, \tau_{y z 4}, \sigma_{z 5}$ and normal strain $\varepsilon_{z 3}$.

We have from constitutive relations

$$
\gamma_{x z 2}=S_{44} \tau_{x z 2}+S_{45} \tau_{y z 2} \Longleftrightarrow(x, y),(4,5) .
$$

Modified displacements and the corresponding derived quantities denoted with $*$ are

$$
u_{3}^{*}=u_{3}-\alpha w_{2, x}+\gamma_{x z 2} \Longleftrightarrow(x, y),(u, v) .
$$

Strain-displacement relations give

$$
\begin{gathered}
\varepsilon_{x 3}^{*}=\varepsilon_{x 3}-\alpha^{2} w_{2, x x}+\alpha \gamma_{x z 2, x} \Longleftrightarrow(x, y), \\
\gamma_{x y 3}^{*}=\gamma_{x y 3}-2 \alpha^{2} w_{2, x y}+\alpha\left(\gamma_{x z 2, y}+\gamma_{y z 2, x}\right), \\
\gamma_{x z 3}^{*}=u_{3}+\gamma_{x z 2} \Longleftrightarrow(x, y),(u, v) .
\end{gathered}
$$

In-plane and transverse shear stresses from constitutive relations are

$$
\begin{gathered}
\sigma_{i(3)}^{*}=Q_{i j} \varepsilon_{j(3)}^{*} \quad(i, j=1,2,3), \\
\tau_{x z 2}^{*}=Q_{44} u_{3}+Q_{45} v_{3}+\tau_{x z 2} \Longleftrightarrow(x, y),(4,5) .
\end{gathered}
$$

Suffix (3) in (39) is to indicate that the stresses and strains correspond to $u_{3}$ and $v_{3}$.

We get from (2), (15), (35), (37), and (40) with reference to coefficient of $f_{3}(z)$ :

$$
\alpha\left[\left(Q_{44} u_{3}+Q_{45} v_{3}\right)_{, x}+\left(Q_{54} u_{3}+Q_{55} v_{3}\right)_{, y}\right]=\beta_{3} \sigma_{z 5} .
$$

Note that $w_{2}$ is not present in the previous equation.

From integration of equilibrium equations, we have with sum on $j=1,2,3$

$$
\begin{aligned}
& \tau_{x z 4}=\alpha\left[Q_{1 j} \varepsilon_{j, x}^{*}+Q_{3 j} \varepsilon_{j, y}^{*}\right]_{(3)}, \\
& \tau_{y z 4}=\alpha\left[Q_{2 j} \varepsilon_{j, y}^{*}+Q_{3 j} \varepsilon_{j, x}^{*}\right]_{(3)} .
\end{aligned}
$$

Note that $w_{2}$ is present in the previous expression.

$$
\begin{gathered}
\alpha\left(\tau_{x z 4, x}+\tau_{y z 4, y}\right)+\sigma_{z 5}=0 \quad\left(\text { Coefficient of } f_{5}\right), \\
\varepsilon_{z 3}=\left[S_{6 j} \sigma_{j}+S_{66} \sigma_{z}\right]_{(3)} .
\end{gathered}
$$

One gets one equation governing in-plane displacements $\left(u_{3}, v_{3}\right)$ from (41), (43), noting that $f_{5, z z}+f_{3}=0$, in the form

$$
\begin{aligned}
& \beta_{3}\left(\tau_{x z 4, x}+\tau_{y z 4, y}\right) \\
& =\alpha^{2}\left[\left(Q_{44} u_{3}+Q_{45} v_{3}\right)_{, x x}+\left(Q_{54} u_{3}+Q_{55} v_{3}\right)_{, y y}\right] .
\end{aligned}
$$

The second equation governing these variables is from the condition $\omega_{z}=0$. Here, it is more convenient to express $\left(u_{3}, v_{3}\right)$ as

$$
\left[u_{3}, v_{3}\right]=-\alpha\left[\left(\psi_{3, x}-\phi_{3, y}\right),\left(\psi_{3, y}+\phi_{3, x}\right)\right]
$$

so that $\Delta \varphi_{3}=0$ and (45) becomes a fourth-order equation governing $\psi_{3}$. This sixth-order system is to be solved for $\psi_{3}$ and $\varphi_{3}$ with associated edge conditions

$$
\begin{array}{lll}
u_{3}^{*}=0 & \text { or } & \sigma_{3}^{*}=0, \\
v_{3}^{*}=0 & \text { or } & \tau_{x y 3}^{*}=0, \\
\psi_{3}=0 & \text { or } & \tau_{x z 3}^{*}=0,
\end{array}
$$

along an $x=$ constant edge and analogous conditions along a $y=$ constant edge.

In (45) expressed in terms of $\psi_{3}$ and $\varphi_{3}$, we replace $w_{2}$ by $\psi_{3}$ for obtaining neutral plane deflection since contributions of $w_{2}$ and $\psi_{3}$ are one and the same in finding $\left[u_{3}, v_{3}\right]$.

7.1. Supplementary Problem. Because of the use of integration constant to satisfy face shear conditions, vertical deflection of neutral plane is equal to that of the face plane deflection. This is physically incorrect since face plane is bounded by elastic material on one side whereas neutral plane is bounded on both sides. This lack in interior solution is rectified by adding the solution of a supplementary problem based on leading cosine term that is enough in view of $f_{2}(z)$ distribution of reactive shear stresses. This is based on the expansion 
of $\sigma_{z}=f_{3}(z)(3 / 2) q$ in sine series. Here, load condition is satisfied by the leading sine term and coefficients of all other sine terms are zero.

Corrective displacements in the supplementary problem are assumed in the form

$$
\begin{gathered}
w=w_{2 s} \frac{\pi}{2} \cos \left(\frac{\pi}{2} z\right), \quad u_{s}=u_{3 s} \sin \left(\frac{\pi}{2} z\right) \Longleftrightarrow(u, v), \\
\sigma_{3 s i}=Q_{i j} \varepsilon_{3 s j} \quad(i, j=1,2,3) .
\end{gathered}
$$

We have from integration of equilibrium equations

$$
\begin{aligned}
& \tau_{2 s s x z}=-\left(\frac{2}{\pi}\right) \alpha\left[Q_{1 j} \varepsilon_{3 s j, x}+Q_{3 j} \varepsilon_{3 s j, y}\right], \\
& \tau_{2 s y z}=-\left(\frac{2}{\pi}\right) \alpha\left[Q_{2 j} \varepsilon_{3 s j, y}+Q_{3 j} \varepsilon_{3 s j, x}\right] .
\end{aligned}
$$

In-plane distributions $u_{3 s}$ and $v_{3 s}$ are added as corrections to the known $u_{3}^{*}$ and $v_{3}^{*}$ so that $(u, v)$ in the supplementary problem are

$$
u=\left(u_{3}^{*}+u_{3 s}\right) \sin \left(\frac{\pi}{2} z\right) \Longleftrightarrow(u, v) .
$$

We note that $\sigma_{z}$ from integration of equilibrium equations with $s$ variables is the same as $\sigma_{z}$ from static equilibrium equations with $*$ variables. Hence,

$$
\left(\frac{2}{\pi}\right)^{2} \alpha^{2}\left[Q_{1 j} \varepsilon_{3 s j, x x}+2 Q_{3 j} \varepsilon_{3 s j, x y}+Q_{2 j} \varepsilon_{3 s j, y y}\right]+\beta_{3} \sigma_{z 5}=0 .
$$

It is convenient to use here also

$$
\left[u_{3 s}, v_{3 s}\right]=-\alpha\left[\left(\psi_{3 s, x}-\phi_{3 s, y}\right),\left(\psi_{3 s, y}+\varphi_{3 s, x}\right)\right] .
$$

Correction to neutral plane deflection due to solution of supplementary problem is from

$$
\varepsilon_{z 3 s}=\left[S_{6 j} \sigma_{3 s j}+S_{66} \sigma_{z 3 s}\right] \quad(\operatorname{sum} j=1,2,3) .
$$

7.2. Summary of Results from the First Stage of Iterative Procedure. The previous analysis gives displacements $[u v]=$ $f_{3}\left[u_{3}, v_{3}\right]$, consistent with $\left[\tau_{x z}, \tau_{y z}\right]=\alpha f_{2}\left[\psi_{, x}, \psi_{, y}\right]$, and corrective transverse stresses from the first stage of iteration

$$
\begin{gathered}
w=w_{0}-\left[f_{2} \varepsilon_{z 1}+f_{4} \varepsilon_{z 3}\right]-\varepsilon_{z s 3} \frac{\pi}{2} \cos \left(\frac{\pi}{2} z\right), \\
u=f_{1} u_{1}+f_{3} u_{3}^{*}+\left(u_{3}^{*}+u_{3 s}\right) \sin \left(\frac{\pi}{2} z\right) \Longleftrightarrow(u, v), \\
\tau_{x z}=f_{2} \tau_{2 x z}+\tau_{2 s x z} \frac{\pi}{2} \cos \left(\frac{\pi}{2} z\right) \Longleftrightarrow(x, y), \\
\sigma_{z}=f_{3}(z) \sigma_{z 3}^{*} \quad(\text { From (35)). }
\end{gathered}
$$

It is to be noted that the dependence of transverse stresses on material constants is through the solution of supplementary problem. One may add $f_{4}$ terms in shear components and $f_{5}$ term in normal stress component. These components are also dependent on material constants, but they need correction from the solution of a supplementary problem. Successive application of the previous iterative procedure leads to the solution of the $3 \mathrm{D}$ problem in the limit.

\section{Concluding Remarks}

Poisson's theory developed in the present study for the analysis of bending of anisotropic plates within small deformation theory forms the basis for generation of proper sequence of 2D problems. Analysis for obtaining displacements, thereby, bending stresses along faces of the plate is different from solution of a supplementary problem in the interior of the plate. A sequence of higher order shear deformation theories lead to solution of associated torsion problem only. In the preliminary solution, reactive transverse stresses are independent of material constants. In view of layer-wise theory of symmetric laminated plates proposed by the present author [10], Poisson's theory is useful since analysis of face plies is independent of lamination which is in confirmation of (8)-(9) in a recent NASA technical publication by Tessler et al. [11].

One significant observation is that sequence of $2 \mathrm{D}$ problems converging to $3 \mathrm{D}$ problems in the analysis of extension, bending, and torsion problems are mutually exclusive to one other.

\section{Highlights}

(i) Poisson-Kirchhoff boundary conditions paradox is resolved.

(ii) Transverse stresses are independent of material constants in primary solution.

(iii) Edge support condition on vertical deflection has no role in the analysis.

(iv) Finding neutral plane deflection requires solution of a supplementary problem.

(v) Twisting stress distribution in pure torsion nullifies its distribution in bending.

\section{Appendix}

$a=$ side length of a square plate

$2 h=$ plate thickness

$f n(z)=$ through-thickness distribution functions, $n=$ $0,1,2 \ldots$

$L=$ characteristic length of the plate in $X-Y$-plane

$q(x, y)=$ applied face load density

$Q_{i j}=$ stiffness coefficients

$S_{i j}=$ elastic compliances

$\left[T_{x}, T_{x y}, T_{x z}\right]=$ prescribed stress distributions along an $x=$ constant edge 
$[U, V, W]=$ displacements in $X, Y, Z$-directions, respectively

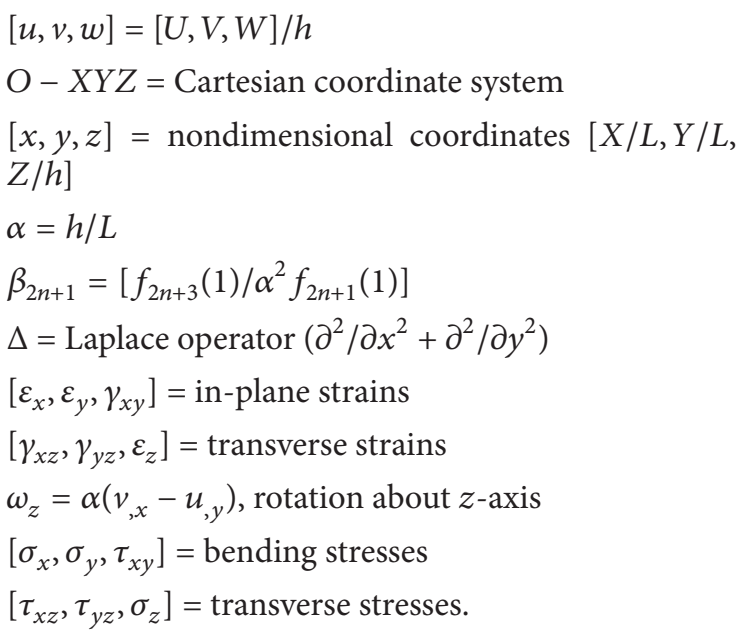

\section{References}

[1] G. Kirchhoff, "Über das Gleichgewicht und die Bewegung einer elastischen Scheibe," Journal für die Reine und Angewandte Mathematik, vol. 40, pp. 51-58, 1850.

[2] H. Hencky, "Über die Berücksichtigung der Schubverzerrung in ebenen Platten," Ingenieur-Archiv, vol. 16, no. 1, pp. 72-76, 1947.

[3] E. Reissner, "Reflections on the theory of elastic plates," Applied Mechanics Reviews, vol. 38, no. 11, pp. 1453-1464, 1985.

[4] A. E. H. Love, A Treatise on Mathematical Theory of Elasticity, Cambridge University Press, Cambridge, UK, 4th edition, 1934.

[5] K. Vijayakumar, "Modified Kirchhoff's theory of plates including transverse shear deformations," Mechanics Research Communications, vol. 38, pp. 211-213, 2011.

[6] K. Vijayakumar, "New look at kirchhoff's theory of plates," AIAA Journal, vol. 47, no. 4, pp. 1045-1046, 2009.

[7] K. Vijayakumar, "A relook at Reissner's theory of plates in bending," Archive of Applied Mechanics, vol. 81, no. 11, pp. 17171724, 2011.

[8] E. Reissner, "The effect of transverse shear deformations on the bending of elastic plates," Journal of Applied Mechanics, vol. 12, pp. A69-A77, 1945.

[9] T. Lewiński, "On the twelfth-order theory of elastic plates," Mechanics Research Communications, vol. 17, no. 6, pp. 375-382, 1990.

[10] K. Vijayakumar, "Layer-wise theory of bending of symmetric laminates with isotropic plies," AIAA Journal, vol. 49, no. 9, pp. 2073-2076, 2011.

[11] A. Tessler, M. Di Sciuva, and M. Gherlone, "Refined zigzag theory for homogeneous, laminated composite, and sandwich plates: a homogeneous limit methodology for zigzag function selection," Tech. Rep. NASA/TP-292010216214:1, 2010. 

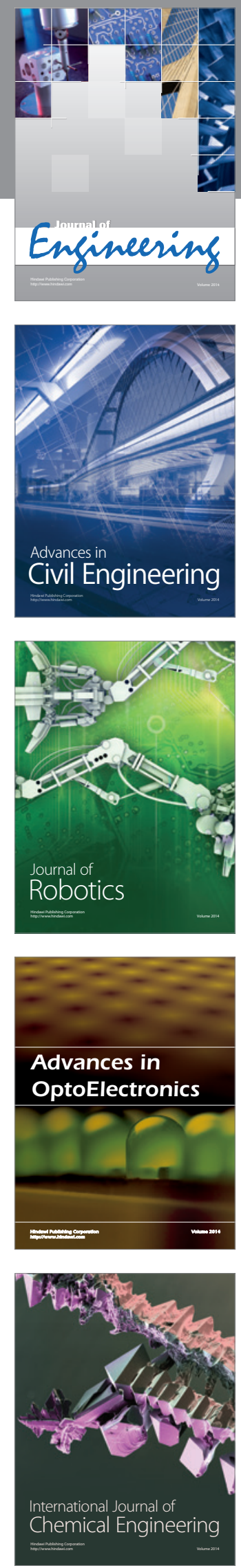

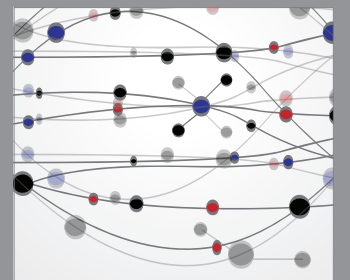

The Scientific World Journal
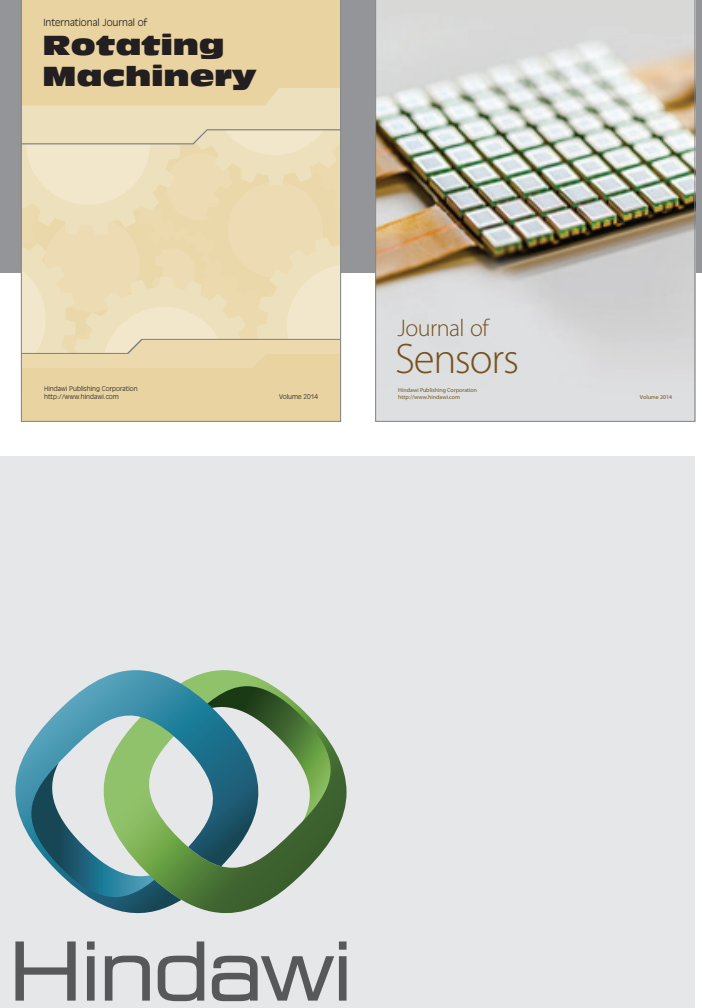

Submit your manuscripts at http://www.hindawi.com
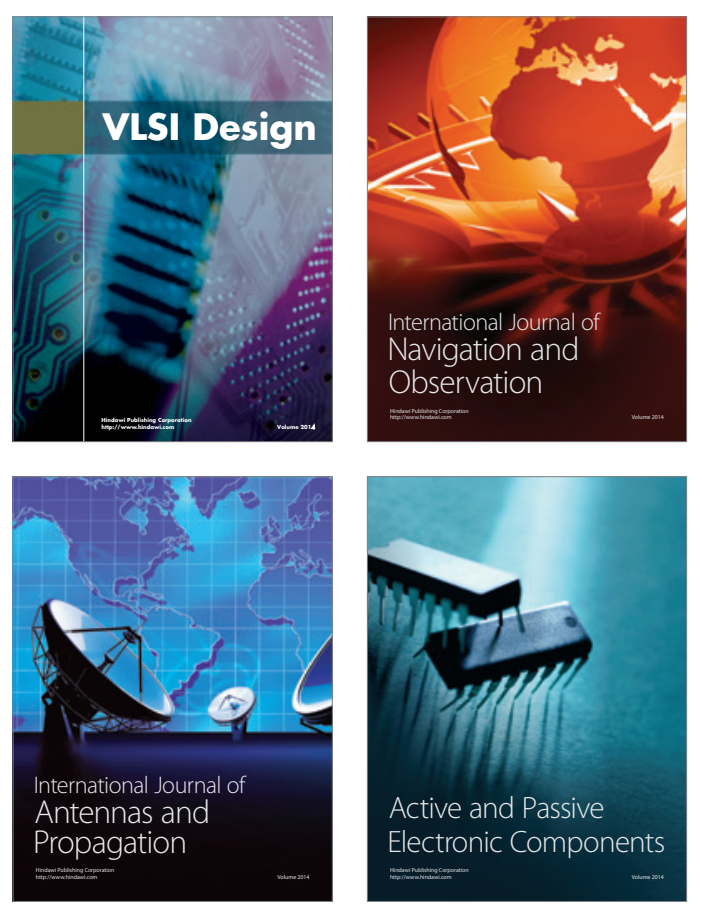
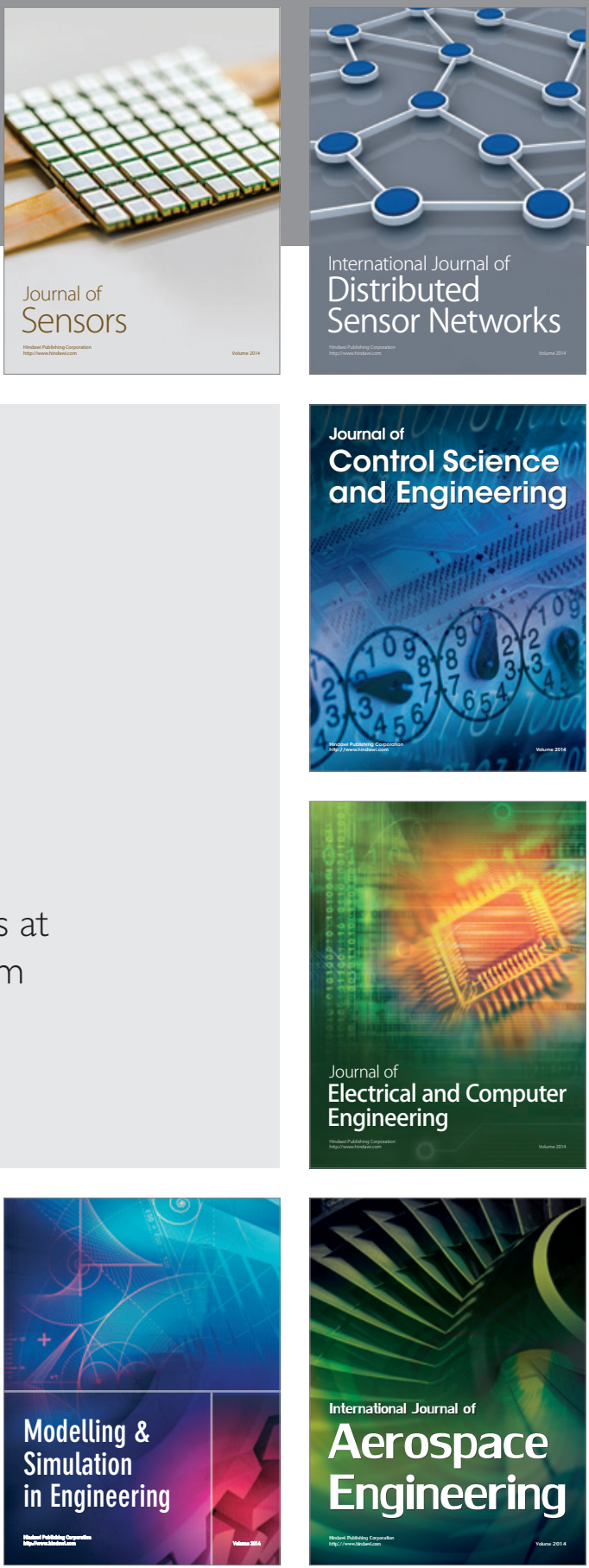

Journal of

Control Science

and Engineering
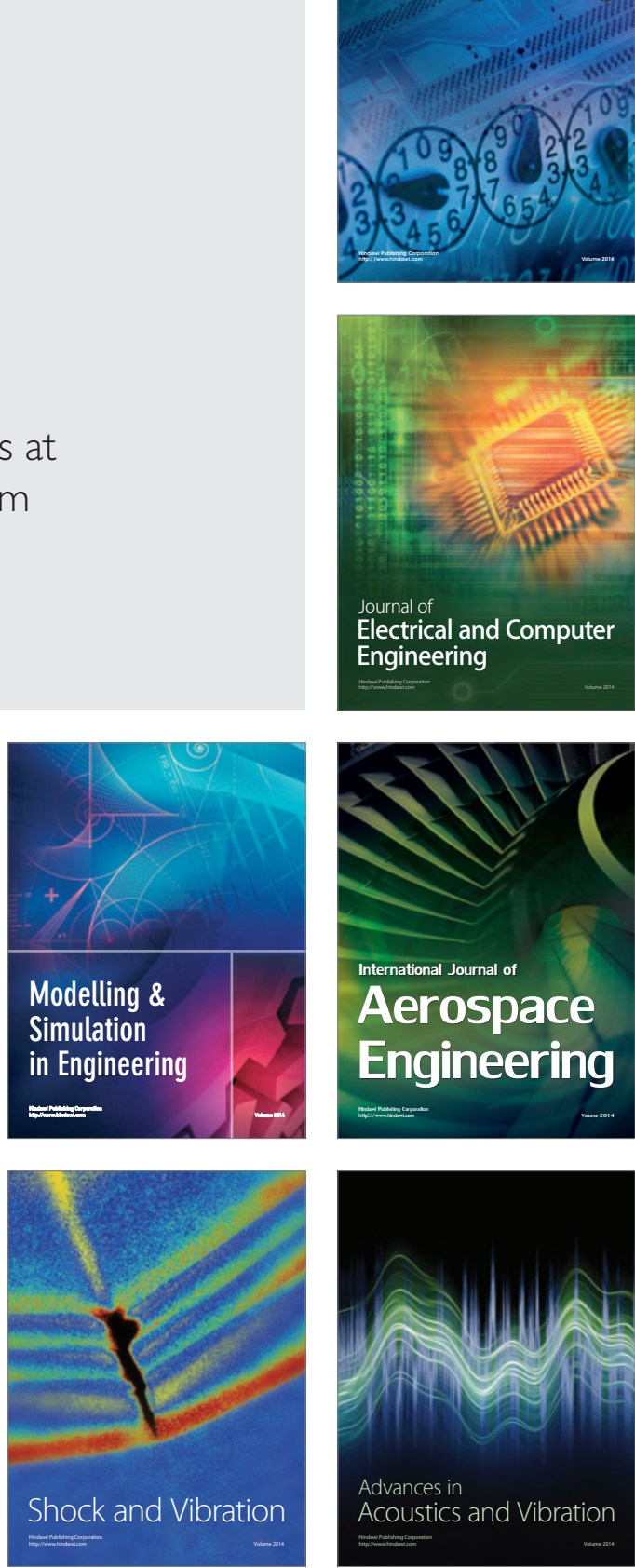